\title{
Drug Delivery Properties of Nanocomposite Particles for Inhalation: Comparison of Drug Concentrations in Lungs and Blood
}

\author{
ISSEI TAKEUCHI $^{1,2}$, YUKI KOSHI $^{1}$ and KIMIKO MAKINO ${ }^{1,2}$ \\ ${ }^{1}$ Faculty of Pharmaceutical Sciences, and ${ }^{2}$ Center for Drug Delivery Research, \\ Tokyo University of Science, Chiba, Japan
}

\begin{abstract}
Background/Aim: Nanocomposite particles are suitable for inhalation; however, their systemic migration has not been confirmed. The aim of this study was to compare drug concentrations in lungs and blood after inhalation of nanocomposite particles. Materials and Methods: Rifampicin (RFP) was used as a model drug. Nanocomposite particles were prepared from dichloromethane with RFP and poly(DLlactic acid-co-glycolic acid) (PLGA) dissolved in an amino acid aqueous solution using a spray dryer. Measurement of RFP concentrations in lung and blood of mice was performed by in vivo tests. Results: Compared with the oral administration group as a control, the RFP concentration in the lungs was significantly higher in the inhalation group. In addition, studies with a fluorescent substance suggested sustained release of drugs from nanocomposite particles in the lungs. Conclusion: Nanocomposite particles deliver pulmonary drug in an efficient and sustained manner.
\end{abstract}

Fine particles have been studied as drug carriers for pulmonary administration (1). The lung has a large surface area and a thin absorption barrier. Local and systemic therapy via the lungs is promising as a drug delivery route due to the low enzyme metabolic activity, the slow mucociliary clearance of the alveoli and the high permeability of the lung epithelia $(2,3)$. Polymer nanoparticles are useful carriers for the delivery of therapeutic agents to the lungs due to their biocompatibility, biodegradability, ease of surface modification, localization, and

This article is freely accessible online.

Correspondence to: Kimiko Makino, Faculty of Pharmaceutical Sciences, Tokyo University of Science, 2641, Yamazaki, Noda, Chiba, 278-8510, Japan. Tel/Fax: +81 471213662, e-mail: makino@rs.noda.tus.ac.jp

Key Words: Nanocomposite particles, nanoparticles, PLGA, inhalation, drug concentration, rifampicin. reduced systemic toxicity (4). They can overcome mucus clearance and become carriers that can efficiently transport drugs to the epithelium, while avoiding undesirable mucociliary clearance (5). While the usefulness of nanoparticles against the A549 lung cancer cell line has been reported (6), nanoparticles need to be formulated for inhalation (7). Their mass median aerodynamic diameter is not suitable for inhalation, and most of the inhaled particles are exhaled, with little particle deposition in the lungs (7-9). In the previous study, we have developed inhalable nanocomposite particles using nanoparticles prepared with poly(DL-lactic acid-co-glycolic acid) (PLGA), a biocompatible polymer $(10,11)$. The nanocomposite particles have an aerodynamic diameter suitable for inhalation. After reaching the alveoli, they are decomposed to nanoparticles by dissolving the diluent amino acids in the alveolar lining fluid (10). The study confirmed the usefulness of arginine and leucine solutions as diluents under high humidity. However, evaluation of drug delivery was performed in an in vitro test using a cascade impactor, and retention of the drug in the lungs and transfer to the blood were not clear. Whether the therapeutic effect of the preparation is local or systemic is important because it greatly affects the method of administration.

The aim of this study was to compare drug concentrations in the lungs and blood when nanocomposite particles were inhaled. Nanocomposite particles were prepared from $\mathrm{O} / \mathrm{W}$ emulsions prepared using PLGA, L-arginine, and L-leucine using spray drying. Rifampicin (RFP), a semi-synthetic bactericidal antibiotic drug, was used as a hydrophobic model drug. The drug is easily quantified using highperformance liquid chromatography (HPLC), and there are already many reports focused on using PLGA to prepare RFP-filled microspheres and nanoparticles (10, 12-15). RFP concentrations in the lungs and blood when nanocomposite particles were administered were confirmed by in vivo experiments using mice. In addition, as a control group, RFP concentrations were measured in the lungs and blood of mice after oral administration of nanocomposite particles. 
Table I. Properties of nanocomposite particles and PLGA nanoparticles (primary particles) (mean \pm S.D., $n=3$ ).

\begin{tabular}{lcc}
\hline & $\begin{array}{c}\text { Nanocomposite } \\
\text { particles }\end{array}$ & $\begin{array}{c}\text { PLGA } \\
\text { nanoparticles }\end{array}$ \\
\hline Mean diameter & $10.9 \pm 14.1 \mu \mathrm{m}$ & $134.6 \pm 35.6 \mathrm{~nm}$ \\
Polydispersity index & - & $0.10 \pm 0.01$ \\
RFP content in particles (\%) & $9.5 \pm 0.3$ & $4.6 \pm 0.3$ \\
Entrapment efficiency (\%) & $47.5 \pm 1.6$ & $23.3 \pm 1.3$ \\
Yield (\%) & $57.6 \pm 1.6$ & - \\
\hline
\end{tabular}

\section{Materials and Methods}

Materials. PLGA with a molecular weight of 10,000 and a DL-lactic acid/glycolic acid monomer composition of $75 / 25$ was purchased from Taki Chemical Co., Ltd. (Kakogawa, Japan). RFP $\left(\mathrm{C}_{43} \mathrm{H}_{58} \mathrm{~N}_{4} \mathrm{O}_{12}\right.$, purity $\geq 97 \%$ ) was purchased from Sigma-Aldrich (St. Louis, MO, USA). 1,1-Dioctadecyl-3,3,3,3-tetramethylindotricarbocyanine iodide (DiR, $\mathrm{C}_{63} \mathrm{H}_{101} \mathrm{IN}_{2}$ ) was purchased from AAT Bioquest, Inc. (Sunnyvale, CA, USA). Isoflurane for the animal was purchased from Mylan Inc. (Pittsburgh, PA, USA). L(+)-arginine (purity $\geq 98 \%$ ), and L-leucine (purity $\geq 99 \%$ ) were purchased from Fujifilm Wako Pure Chemical Corp. (Osaka, Japan). All other chemicals were of the highest grade commercially available.

Preparation of nanocomposite particles. The dispersed phase was prepared by dissolving $100 \mathrm{mg}$ RFP and $400 \mathrm{mg}$ PLGA in $10 \mathrm{ml}$ dichloromethane. The solution was added to $50 \mathrm{ml}$ of aqueous solution in which $500 \mathrm{mg}$ of amino acid (arginine: leucine=1: 6) was dissolved. The mixed solution was emulsified using a probe sonicator (Digital Sonifier S-250D, Branson Ultrasonics Corp. Danbury, CT, USA) for $20 \mathrm{sec}$ at $200 \mathrm{~W}$ of energy output. Then, the $\mathrm{O} / \mathrm{W}$ emulsion was spray-dried to prepare nanocomposite particles using a spray dryer (Mini Spray Dryer B-290, BÜCHI corp., Flawil, Switzerland) under the outlet temperature of $37-40^{\circ} \mathrm{C}$, the air volume of $22.5 \mathrm{~m}^{3} / \mathrm{h}$ and solution sending speed of $1.2 \mathrm{ml} / \mathrm{min}(7$, 10). After freezing at $-30^{\circ} \mathrm{C}$, nanocomposite particles were lyophilized using a freeze dryer (FD-1000, Tokyo Rikakikai Co., Ltd., Tokyo, Japan) for $12 \mathrm{~h}(10,14)$.

The size of nanocomposite particles in the air was measured by using a sizer (LDSA-3500A, Nikkiso Co., Ltd., Tokyo, Japan). The mean volume diameters of RFP-loaded PLGA nanoparticles redispersed from the nanocomposite particles were measured using a particle size analyzer (ELSZ-1000ZS) at $25^{\circ} \mathrm{C}$. RFP contents in the nanocomposite particles were measured using HPLC (SIL-20A prominence, SPD-20A prominence, LC-20AD prominence, CTO10ASvp, DGU-20A3 prominence, Shimadzu Co., Kyoto, Japan) at $254 \mathrm{~nm}$ with an ODS column (STR ODS-M, size: $4.6 \mathrm{~mm} \times 150 \mathrm{~mm}$, Shinwa Chemical Industries Ltd., Kyoto, Japan). The mobile phase consisted of phosphate buffer solution ( $\mathrm{pH} 2.6)$ and acetonitrile with a volume ratio of $2: 3$. Five milligrams of the samples were dissolved in $10 \mathrm{ml}$ of the mobile phase. In addition, $30 \mathrm{mg}$ of RFP were dissolved in $10 \mathrm{ml}$ of the solution as a control. HPLC measurements were carried out at $40^{\circ} \mathrm{C}$ (flow rate: $1.0 \mathrm{ml} / \mathrm{min}$ ), and $50 \mu \mathrm{l}$ of sample solution were applied. All HPLC measurements were carried out under the same conditions. RFP contents in PLGA nanoparticles were also measured using HPLC. Briefly, $45 \mathrm{mg}$ of

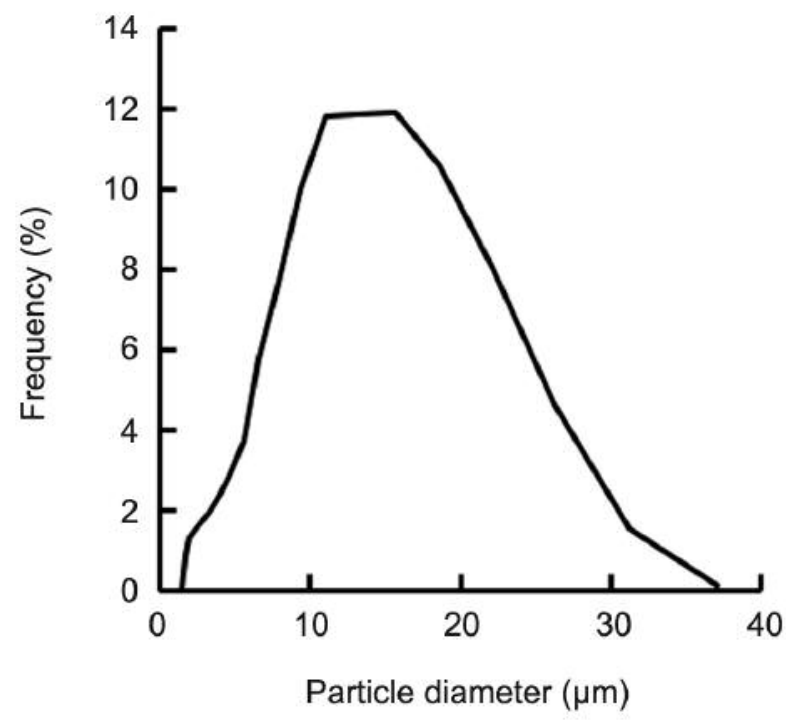

Figure 1. Tize distributions of nanocomposite particles $(n=3)$.

nanocomposite particles were dispersed in $45 \mathrm{ml}$ of purified water, and diluents were removed using a dialysis membrane. After dialysis for 2 days, the solution in the dialysis membrane was lyophilized to obtain a powder sample for HPLC measurement. Surface properties of nanocomposite particles were observed using a scanning electron microscope (SEM, JSM-6060LA, JEOL Ltd., Akishima, Japan).

Evaluation of RFP concentration in mouse lungs and blood. Eightweek-old male ICR mice were purchased from Japan SLC Inc. (Tokyo Japan). All animal care was conducted under the Guidelines for Animal Experimentation of Tokyo University of Science, which are based on the Guidelines for Animal Experimentation of the Japanese Association for Laboratory Animal Science. Mice were housed in stainless-steel cages under standard environmental conditions $\left(23 \pm 1^{\circ} \mathrm{C}, 55 \% \pm 5 \%\right.$ humidity and a $12 / 12 \mathrm{~h} \mathrm{light/dark}$ cycle) and maintained with free access to water and a standard laboratory diet (carbohydrates $30 \%$; proteins $22 \%$; lipids $12 \%$; vitamins 3\%) ad libitum (Nihon Nosan Kogyo Co., Yokohama, Japan). Mice were administered nanocomposite particles under anesthesia with isoflurane. In the pulmonary administration group, $3 \mathrm{mg}$ of nanocomposite particles were administered pulmonary using an insufflator (DPI, Model DP-4M, Penn-Century, Inc., Glenside, PA, USA). In the oral administration group, a control group, $3 \mathrm{mg}$ of $\mathrm{NC}$ particles were dissolved in $1 \mathrm{ml}$ of physiological saline and administered into the stomach. RFP concentrations in mouse lungs and blood were measured using HPLC. After 0.25, 0.5, $1,2,4,8$, and $12 \mathrm{~h}$ from initiation of the test, mice were euthanized with isoflurane and $700 \mu \mathrm{l}$ of blood were collected. Then the lungs were removed and washed with saline. The lungs were divided into 5 lobes and tissue samples were homogenized in $200 \mu$ l of physiological saline. To remove protein, $800 \mu \mathrm{l}$ of ethanol and acetonitrile were added to lung tissue and blood, respectively. After centrifuging the sample solution, $600 \mu$ of the collected supernatant was dried using nitrogen gas to obtain a sample for HPLC measurement. 


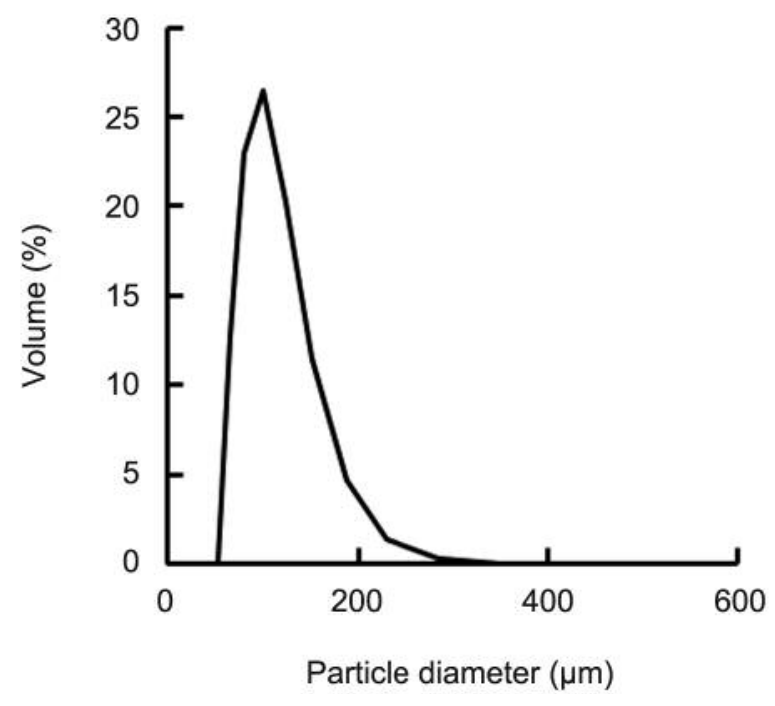

Figure 2. Size distributions of PLGA nanoparticles ( $n=3)$.

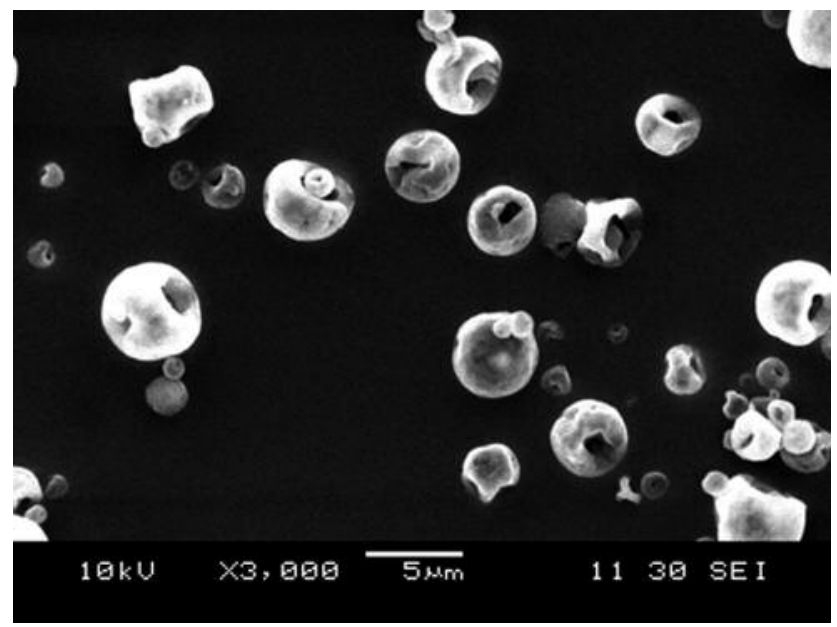

Figure 3. Scanning electron microscopy images of nanocomposite particles taken at an accelerating voltage of $10 \mathrm{kV}$ (magnification: $3,000 \times)$.
The retentivity of the nanocomposite particles in mouse lungs was confirmed using an in vivo optical imaging system (Clairvivo OPT plus, Shimadzu Corp.). Nanocomposite particles containing $\mathrm{DiR}$, a fluorescent substance, were prepared in the same manner as the nanocomposite particles using $400 \mathrm{mg}$ of PLGA, $40 \mathrm{mg}$ of RFP, $10 \mathrm{mg}$ of DiR. Mice (8 years old, ICR, male) were anesthetized with isoflurane, and $3 \mathrm{mg}$ of nanocomposite particles containing DiR were administered pulmonary and orally in the same manner as described above. Immediately after administration, and after 2, 4, and $8 \mathrm{~h}$ from administration, evaluation of pulmonary retention of nanocomposite particles was carried out using an in vivo optical imaging system. The measurement was performed with an exposure time of $1 \mathrm{sec}$, an excitation wavelength of $735 \mathrm{~nm}$, and a fluorescence wavelength of $800 \mathrm{~nm}$. For $6 \mathrm{~h}$ prior measurement, mice had limited food access (16).

\section{Results}

We prepared nanocomposite particles with a mean diameter of $10.9 \pm 14.1 \mu \mathrm{m}$, which contained PLGA nanoparticles with a mean diameter of $134.6 \pm 35.6 \mathrm{~nm}$. Their RFP content and RFP entrapment efficiency are summarized in Table I. RFP contents in nanocomposite particles and PLGA nanoparticles were 9.5 and $4.6 \%$, respectively. This result indicates that $48 \%$ of the RFP contained in the nanocomposite particles was present in the PLGA nanoparticles. Drugs contained in PLGA nanoparticles can be expected to have sustained release properties (10). The size distributions of the nanocomposite particles and the nanoparticles are shown in Figures 1 and 2, respectively. Figure 3 displays a SEM image of the nanocomposite particles. It was confirmed that the nanocomposite particles prepared from the $\mathrm{O} / \mathrm{W}$ emulsion were non-spherical. From these findings, we confirmed that non-spherical nanocomposite particles for inhalation were prepared successfully.

In the in vivo study using mice, no RFP was detected in the blood at all times when nanocomposite particles were administered orally. Figure 4 shows the RFP concentrations in the lung and blood when the particles were administered by inhalation, and the concentration in the lungs when administered orally. At $0.25-12 \mathrm{~h}$ after nanocomposite particle administration, the RFP concentration in the lungs after inhalation was significantly higher than that in the other two. Following inhalation, the RFP concentration in the blood was detected at all times measured and ranged from 0.13 to 0.01 $\mu \mathrm{g} / \mathrm{ml}$. Following oral administration, the RFP concentration in the lungs was only detected at 2 and $4 \mathrm{~h}$ after administration, and was significantly higher than that in the blood at $4 \mathrm{~h}$ after inhalation of the nanocomposite particles.

Figure 5 shows the retentivity of the nanocomposite particles in mouse lungs. When nanocomposite particles were inhaled, DiR-derived fluorescence was observed in the lungs at $0-8 \mathrm{~h}$. When comparing the fluorescence intensity, it was revealed that the fluorescence was strongest at $2 \mathrm{~h}$. In contrast, when nanocomposite particles were administered orally, no fluorescence in the lung was observed at 0-8 h.

\section{Discussion}

In the SEM image (Figure 1), the average particle size of the non-spherical nanocomposite particles was approximately 2$5 \mu \mathrm{m}$. We considered that the particle diameter was different from that measured using a laser diffraction size analyzer due to the shape of these particles (14). As shown in Figure 


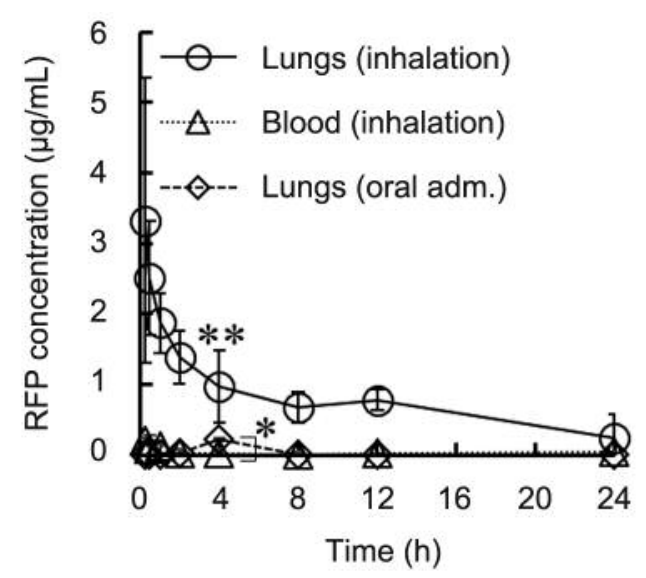

Figure 4. RFP concentrations in mouse lungs and blood when the particles were administered by inhalation [lungs (inhalation) and blood (inhalation)], and the concentration in the lungs when administered orally [lungs (oral adm)] (mean \pm S.D., $n=3, * p<0.05$, **p<0.01, Tukey's test).

4, nanocomposite particles are useful for drug delivery to the lung. At $0.25 \mathrm{~h}$ after inhalation, the RFP concentration in the lung was 27.2 times higher compared to that in the blood, and after $12 \mathrm{~h}$ it was 37.7 times higher. A small amount of RFP was detected in the lung at 2 and $4 \mathrm{~h}$ after oral administration, however, its retention was low. From these results, we considered that the drug contained in the nanocomposite particles had a high local action in the lung. It is suggested that inhalation of nanocomposite particles may have reduced side effects because it showed significantly higher drug concentrations in the lung when inhaled, despite the low drug dose that could not be detected in the blood when administered orally. The maximum fluorescence derived from $\mathrm{DiR}$ at $2 \mathrm{~h}$ after inhalation suggests the release of DiR from PLGA nanoparticles (Figure 5). We considered that immediately after inhalation of the nanocomposite particles, DiR in the diluent and on the surface of the PLGA nanoparticles showed fluorescence, and the fluorescence intensity increased after $2 \mathrm{~h}$ because DiR released from PLGA nanoparticles was taken up by cells and showed fluorescence. These results indicate a sustained release of the drug from the nanocomposite particles.

\section{Conclusion}

In this study, nanocomposite particles were prepared from an $\mathrm{O} / \mathrm{W}$ emulsion using a spray dryer. From the results of in vivo studies, we confirmed that inhalation of nanocomposite particles can deliver drugs to the lungs more efficiently than oral administration. In addition, it was suggested that nanocomposite particles have sustained drug release in the lung. These results can help reduce side effects in drug

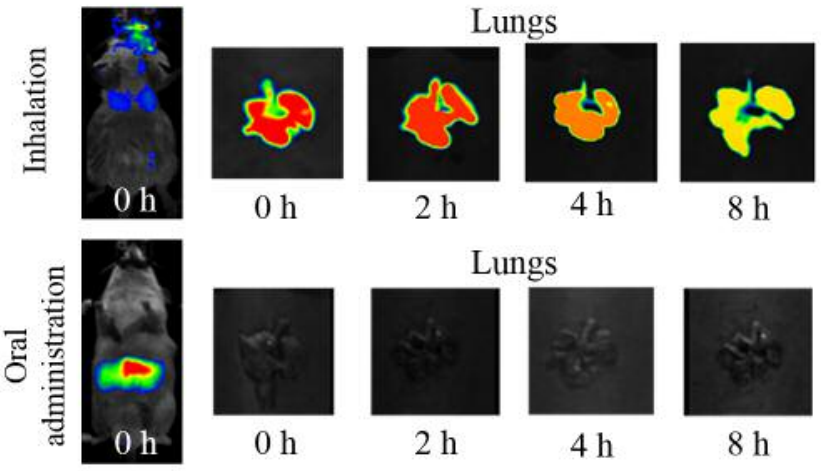

Figure 5. Fluorescence image of mouse lungs observed using an in vivo optical imaging system when inhaling nanocomposite particles containing DiR.

therapy targeting the lung. Further studies on methods for preparing nanocomposite particles via $\mathrm{O} / \mathrm{W}$ emulsion and research on drug release behavior from the particles will contribute to the development of inhalation formulations.

\section{Conflicts of Interest}

The Authors declare that they have no potential conflicts of interest regarding this study.

\section{Authors' Contributions}

I. Takeuchi and Y. Koshi designed the study, and wrote the initial draft of the manuscript. K. Makino contributed to analysis and interpretation of data, and assisted in the preparation of the manuscript. All Authors approved the final version of the manuscript, and agreed to be accountable for all aspects of the work in ensuring that questions related to the accuracy or integrity of any part of the work are appropriately investigated and resolved.

\section{Acknowledgements}

The Authors are grateful for the suggestions given by $\mathrm{Mr}$. T. Nakajima from Tokyo University of Science (Center for Drug Delivery Research).

\section{References}

1 Paranjpe M and Müller-Goymann CC: Nanoparticle-mediated pulmonary drug delivery: a review. Int J Mol Sci 15: 5852-5873, 2014. PMID: 24717409. DOI: 10.3390/ijms15045852

2 Köhler D: Aerosols for systemic treatment. Lung 168: 677-684, 1990. PMID: 2117179. DOI: 10.1007/bf02718194

3 Yamamoto H, Kuno Y, Sugimoto S, Takeuchi H and Kawashima Y: Surface-modified PLGA nanosphere with chitosan improved pulmonary delivery of calcitonin by mucoadhesion and opening of the intercellular tight junctions. J Control Release 102: 373381, 2005. PMID: 15653158. DOI: 10.1016/j.jconrel.2004.10.010 
4 Menon JU, Ravikumar P, Pise A, Gyawali D, Hsia CC and Nguyen KT: Polymeric nanoparticles for pulmonary protein and DNA delivery. Acta Biomater 10: 2643-2652, 2014. PMID: 24512977. DOI: $10.1016 /$ j.actbio.2014.01.033

5 Schürch S, Gehr P, Im Hof V, Geiser M and Green F: Surfactant displaces particles toward the epithelium in airways and alveoli. Respir Physiol 80: 17-32, 1990. PMID: 2367749. DOI: 10.1016/0034-5687(90)90003-h

6 Huang M, Ma Z, Khor E and Lim LY: Uptake of FITC-chitosan nanoparticles by A549 cells. Pharm Res 19: 1488-1494, 2002. PMID: 12425466. DOI: 10.1023/a:1020404615898

7 Tomoda K, Ohkoshi T, Kawai Y, Nishiwaki M, Nakajima T and Makino K: Preparation and properties of inhalable nanocomposite particles: effects of the temperature at a spraydryer inlet upon the properties of particles. Colloids Surf B Biointerfaces 61: 138-144, 2008. PMID: 17890065. DOI: 10.1016/j.colsurfb.2007.07.016

8 Finlay WH, Stapleton KW and Zuberbuhler P: Fine particle fraction as a measure of mass depositing in the lung during inhalation of nearly isotonic nebulized aerosols. J Aerosol Sci 28: 1301-1309, 1997. DOI: 10.1016/S0021-8502(97)00017-7

9 Finlay WH and Gehmlich MG: Inertial sizing of aerosol inhaled from two dry powder inhalers with realistic breath patterns versus constant flow rates. Int J Pharm 210: 83-95, 2000. PMID: 11163990. DOI: 10.1016/s0378-5173(00)00569-x

10 Takeuchi I, Tetsuka Y, Nii T, Shinogase M and Makino K: Inhalable nanocomposite particles using amino acids with improved drug content and humidity resistance. Colloids Surf A 529: 387-393, 2017. DOI: 10.1016/j.colsurfa.2017.06.003

11 Ignatius $\mathrm{AA}$ and Claes LE: In vitro biocompatibility of bioresorbable polymers: poly(L, DL-lactide) and poly(L-lactideco-glycolide). Biomaterials 17: 831-839, 1996. PMID: 8730968. DOI: $10.1016 / 0142-9612(96) 81421-9$
$12 \mathrm{Hu} \mathrm{C}$, Feng $\mathrm{H}$ and Zhu C: Preparation and characterization of rifampicin-PLGA microspheres/sodium alginate in situ gel combination delivery system. Colloids Surf B Biointerfaces 95: 162-169, 2012. PMID: 22424828. DOI: 10.1016/j.colsurfb. 2012.02.030

13 Doan TV, Couet $\mathrm{W}$ and Olivier JC: Formulation and in vitro characterization of inhalable rifampicin-loaded PLGA microspheres for sustained lung delivery. Int J Pharm 414: 112117, 2011. PMID: 21596123. DOI: 10.1016/j.jpharm.2011.05.007

14 Takeuchi I, Taniguchi Y, Tamura Y, Ochiai K and Makino K: Effects of 1-leucine on PLGA microparticles for pulmonary administration prepared using spray drying: Fine particle fraction and phagocytotic ratio of alveolar macrophages. Colloids Surf A 537: 411-417, 2018. DOI: 10.1016/j.colsurfa.2017.10.047

15 Nii T, Takeuchi I, Kimura Y and Makino K: Effects of the conformation of PLGA molecules in the organic solvent on the aerodynamic diameter of spray dried microparticles. Colloids Surf A 539: 347-353, 2018. DOI: 10.1016/j.colsurfa.2017.12.042

16 Takeuchi I, Kamiki Y and Makino K: Therapeutic efficacy of rebamipide-loaded PLGA nanoparticles coated with chitosan in a mouse model for oral mucositis induced by cancer chemotherapy. Colloids Surf B Biointerfaces 167: 468-473, 2018. PMID: 29723818. DOI: 10.1016/j.colsurfb.2018.04.047

Received December 4, 2019

Revised December 11, 2019

Accepted December 13, 2019 